Jianjun Zhang, Zhihua Wang* and Longmao Zhao

\title{
Cell wall material strain hardening on dynamic responses of closed-cell foams
}

DOI 10.1515/secm-2015-0193

Received April 11, 2015; accepted April 2, 2016; previously published online May 3, 2016

\begin{abstract}
The paper focuses on the effects of the cell wall material strain hardening on the mechanical behavior of closed-cell foams subjected to the constant velocity loading. Three-dimensional Voronoi models were established to present the closed-cell foams, and the simulations were performed by employing Ls-dyna 971. The investigation found that the cell wall strain hardening could postpone the onset of the localized collapse bands and retard deformation mode transformation. Strain hardening convergence phenomenon was observed in the closed-cell foams; increasing the impact velocity could weaken the effect of the material strain hardening. The crushing stress at both impact and stationary sides were analyzed in detail. The material strain hardening could always enhance the plastic energy dissipation. Furthermore, the strain hardening convergence was also observed in the plateau stress at the impact side and plastic energy dissipation but not in the densification strain and the plateau at the stationary side.
\end{abstract}

Keywords: cellular material; impact loading; strain hardening convergence; 3D Voronoi.

\section{Introduction}

Cellular materials offer the ability to undergo large plastic deformation at nearly constant nominal stress and thus can absorb a large amount of kinetic energy before collapsing into a more stable configuration or fracture. These

\footnotetext{
*Corresponding author: Zhihua Wang, Institute of Applied Mechanics and Biomedical Engineering, Taiyuan University of Technology, Taiyuan 030024, China, Phone: +8603516010560 , e-mail:wangzh@tyut.edu.cn Jianjun Zhang and Longmao Zhao: Institute of Applied Mechanics and Biomedical Engineering, Taiyuan University of Technology, Taiyuan 030024, China
}

unique mechanical properties make them popular candidates for a wide range of applications including packaging, shock mitigation and myriad aerospace applications [1]. Considerable experimental and numerical studies have been conducted to investigate the dynamic behavior of cellular materials [1-9].

Inelastic properties of the material can add significant complexity to the mechanical response of the cellular materials. Papka and Kyriakides [10] previously took the effects of the strain hardening of the cell wall material of cellular structures into consideration using a combination of experiments and analyses. Some others [11-14] studied the effect of the material strain hardening on the response of the open-cell foams at a quasi-static case. Mangipudi et al. [15] elucidated the micro-structural origin of strain hardening in open-cell metal foams and developed a multi-scale model to study the development of plasticity at two length scales: (i) the development of plastic zones inside individual struts (microscopic scale) and (ii) the formation of plastic localization bands at the scale of the cellular architecture (mesoscopic scale). They investigated how plasticity at both scales contributed to the macroscopic yielding and strain hardening of cellular metals. Mousanezhad et al. [16] highlighted the effects of cell wall material strain hardening and density functional gradation on in-plane crushing response of hexagonal honeycombs and found that there was a plasticity and gradient convergence phenomenon. Song et al. [17] explored the behavior of closed-cell wall foams. It was found that the strain hardening of the cell wall material had a strengthening effect on the plateau stress and the densification strain energy. The stronger the strain hardening was, the larger the plateau stress and the densification strain energy were. Limited investigations and analyses center on effects of cell wall strain hardening on the closed-cell foams, so in this work, a three-dimensional Voronoi model was established to present the closed-cell foam. The dynamic response of the closed-cell foams with cell wall strain hardening was systematically analyzed. The influence of material strain hardening on the deformation, crushing stress and strain, and plastic energy dissipation were taken into consideration. 


\section{Methodology}

\subsection{Three-dimensional random Voronoi technique}

Three-dimensional Voronoi models are created by randomly placing a set of $N$ nuclei inside a cube with edge length $L$. It must be emphasized that in order to prevent very small cells in the Voronoi models, a subsequent nucleus is accepted into the cube only when its distance to any existing nucleus is greater than $\delta(\delta \geq 0)$. The nucleus generation process continues until no more nuclei can be accepted in the cube. The total number of nuclei in the cube depends on the ratio $\delta / L$. For each nucleus, around it there is a region consisting of all locations in the space which is closer to the nucleus than any other nucleus. The space can then be divided into $N$ cells [18]. The boundaries of all cells constitute the Voronoi diagram, and then, a Voronoi model with periodic boundary cut from the Voronoi structure is obtained.

To build a regular tetrakaidecahedral foam model with $N$ cells in the volume $V_{0}$, the distance between any two adjacent nuclei is given by Weaire and Fortes [19]:

$$
d_{0}=\frac{\sqrt{6}}{2}\left(\frac{V_{0}}{\sqrt{2} N}\right)^{\frac{1}{3}}
$$

To construct a three-dimensional Voronoi foam model with $N$ cells in the volume $V_{0}$, a dimensionless variable $k$ is defined as the degree of irregularity of the porous material.

$$
k=1-\frac{\delta}{d_{0}}
$$

where $\delta$ is the minimum distance between any two nuclei [20].

\subsection{Finite element models}

The Voronoi diagram is similar to the physical structure of cellular materials. In the paper, 3D Voronoi diagrams with the uniform cell wall thickness and cell shape irregularity degrees $k=0.5$ were constructed to present the closed-cell foams; as shown in Figure 1, the size of the model was $20 \mathrm{~mm} \times 20 \mathrm{~mm} \times 20 \mathrm{~mm}$. The simulations were carried out by employing Ls-dyna 971, and shell elements were used for the cell walls [10, 17, 21, 22]. Mesh convergence study was also performed in order to remove any mesh dependence from the final results, and the model with 43,861 elements and 41,512 nodes was

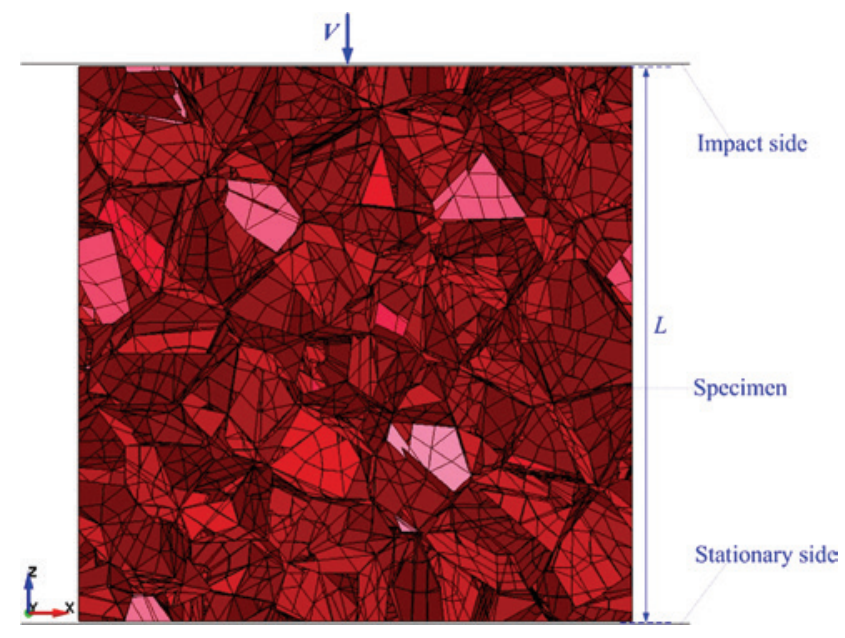

Figure 1: Illustration of the FE model with loading conditions.

adopted in the simulations below. The properties of the cell wall material were selected to be those of aluminum alloy. The density $\left(\rho_{s}\right)$, Young's modulus $(E)$, Poisson's ratio and yield stress $\left(\sigma_{y}\right)$ were assigned as $2.7 \times 10^{3}$ $\mathrm{kg} / \mathrm{m}^{3}, 69 \mathrm{GPa}, 0.33$ and $76 \mathrm{MPa}$, respectively. The bilinear strain-hardening model material was employed for the model, as in the following:

$$
\sigma= \begin{cases}E \varepsilon, & \varepsilon<\varepsilon_{y} \\ \sigma_{y}+H\left(\varepsilon-\varepsilon_{y}\right), & \varepsilon \geq \varepsilon_{y}\end{cases}
$$

here, $\sigma$ and $\varepsilon$ are the stress and strain, and $H$ and $\varepsilon_{y}$ are the hardening tangent modulus and yield strain of the cell wall material. Also, the behavior of the cell wall material was treated as rate independent, as was done in references [6, 7, 17, 21-23]. For the comparison, a dimensionless strain hardening parameter is defined as $\bar{H}=H / E$. The relative density $\left(\rho_{0}=\rho^{\star} / \rho_{s}, \rho^{\star}\right.$ is the density of the specimen) is firstly kept at $6 \%$.

The models in the simulation were sandwiched between an impact rigid plate and a stationary rigid plate, which was similar to the real conditions in the impact test of cellular materials. During the crushing in the $z$ direction, the top plate moved downward with a constant velocity, while the bottom plate was kept stationary. Firstly, the automatic surface-to-surface contact was employed between the top plate and the Voronoi model, as well as between the Voronoi model and the bottom plate. The automatic single-surface contact was defined to all the cell surfaces. Noticeably, the contact edges of foams can slip on both rigid plates with little friction. The dynamic friction coefficient was assumed to be 0.2 . There is no sensitivity found in the simulation results to the friction coefficient by parametric study [24]. 


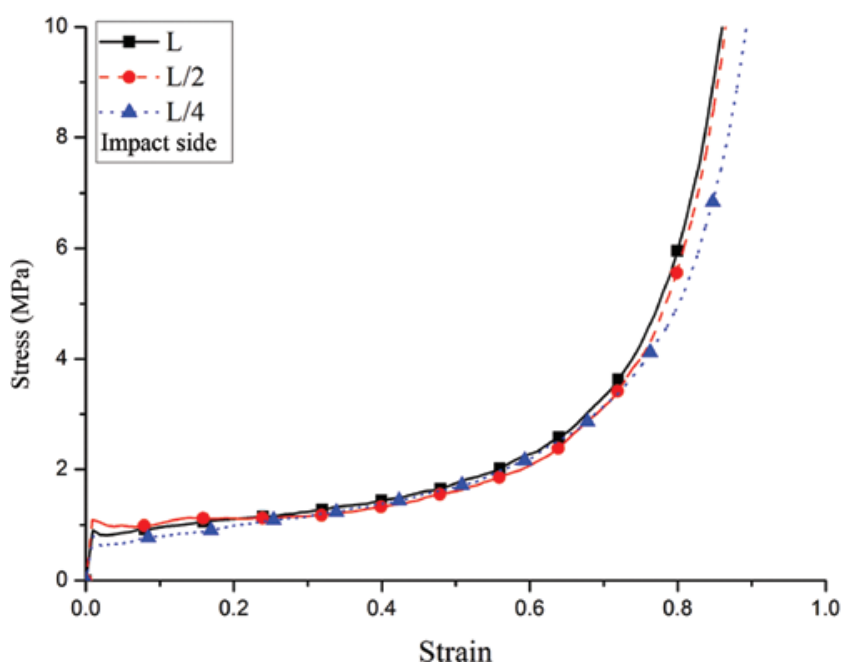

Figure 2: Nominal stress-strain curves at both sides with $V=10 \mathrm{~m} / \mathrm{s}$.

\section{Results and discussion}

Firstly, the nominal stress-strain curve may be different when the specimen size is different. We keep the width of the specimen $20 \mathrm{~mm}$ square and divide it equally into two through the thickness $(L=10 \mathrm{~mm})$ and then further divide it into four equal thin specimens $(L=5 \mathrm{~mm})$. The thin specimen contains insufficient cells to statistically represent the cellular material. All the four thin specimens are simulated, and the average nominal stress-strain curve is presented. Figure 2 presents the results at both sides with $\bar{H}=0.01$. There is negligible difference between the outcomes of these three specimen sizes, which agrees with the findings in reference [24]; the crushing stress at both sides has no appreciable dependency on the nominal strain rate. Thus, the specimen with size $L$ is employed in the following simulations.

We study the effect of the cell wall material strain hardening on the dynamic crushing of the closed-cell foams. Firstly, a dimensionless velocity $(\bar{V})$ is defined as

$$
\bar{V}=V / c_{p}
$$

here, $V$ is the constant velocity, and $c_{p}$ is a characteristic velocity [25] which can be calculated from

$$
c_{p}=\sqrt{\sigma_{0} / \rho^{\star}}
$$

$\sigma_{0}$ is the plateau stress of the specimen; for the closed-cell foams, this can be described as $[1,17]$

$$
\sigma_{0} / \sigma_{y}=0.44\left(\rho^{\star} / \rho_{s}\right)
$$

The characteristic velocity is calculated as about $111 \mathrm{~m} / \mathrm{s}$. Another dimensionless parameter, plastic energy dissipation $\bar{E}_{p}$, is also adopted as

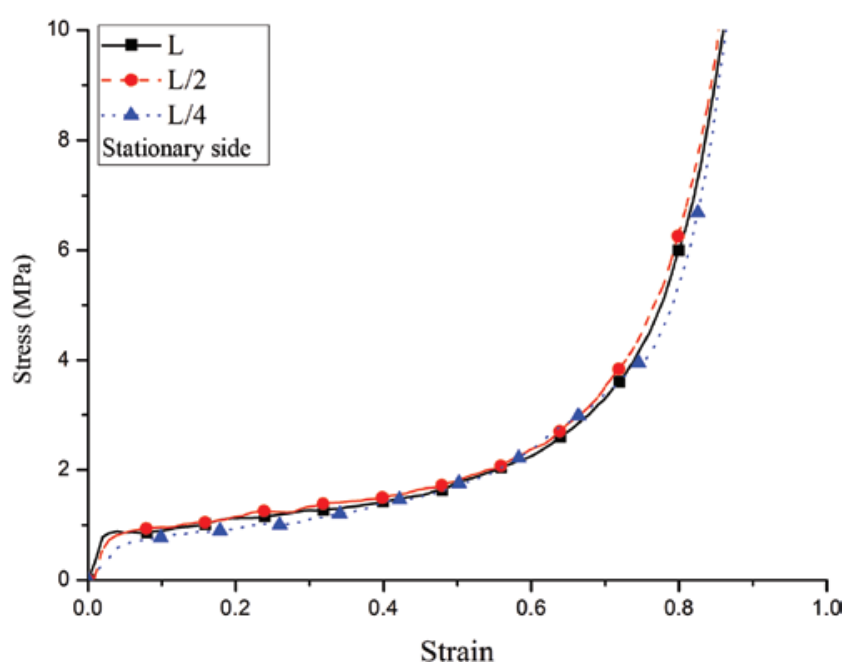

$\bar{E}_{p}=E_{p} / \sigma_{0} A L$

$E_{p}$ is the plastic energy dissipation extracted directly from the finite element (FE) results, $A$ is the cross-sectional area of the specimen normal to $z$ direction, and $L$ is the height of the specimen in the $z$ direction.

\subsection{Deformation mode}

By employing the honeycomb or Voronoi models, references $[6,17,21,23,24,26,27]$ investigated the deformation mode of the cellular material. For the uniform cellular material, there were three macroscopic deformation modes (X-mode, V-mode and I-mode) at different velocity impact. The macroscopic deformation of Voronoi structures is complicated, but it can be classified into three modes [23, 24, 27]; however, they used different names for this classification. Considering this primary effect of the impact velocity, Zheng et al. [23] catalogued the deformation into the Quasi-static mode, Transitional mode and Dynamic mode. Based on the numerical simulation results, Ma et al. [24] detected three deformation modes, namely, Random mode, Transitional mode and Progressive mode, corresponding to increasing impact velocities. Liu et al. [27] named the three deformation modes as the Homogeneous Mode, the Transitional Mode and the Shock Mode. But introducing the hardening parameter may have some effect on the deformation patterns of closed cell foams, so in this section, we probe this influence in detail. Figure 3 shows the deformation patterns at the $50 \%$ crushing strain. For the model with no strain hardening in Figure 3A, three deformation modes can be clearly observed: Quasi-static mode, Transitional mode and 

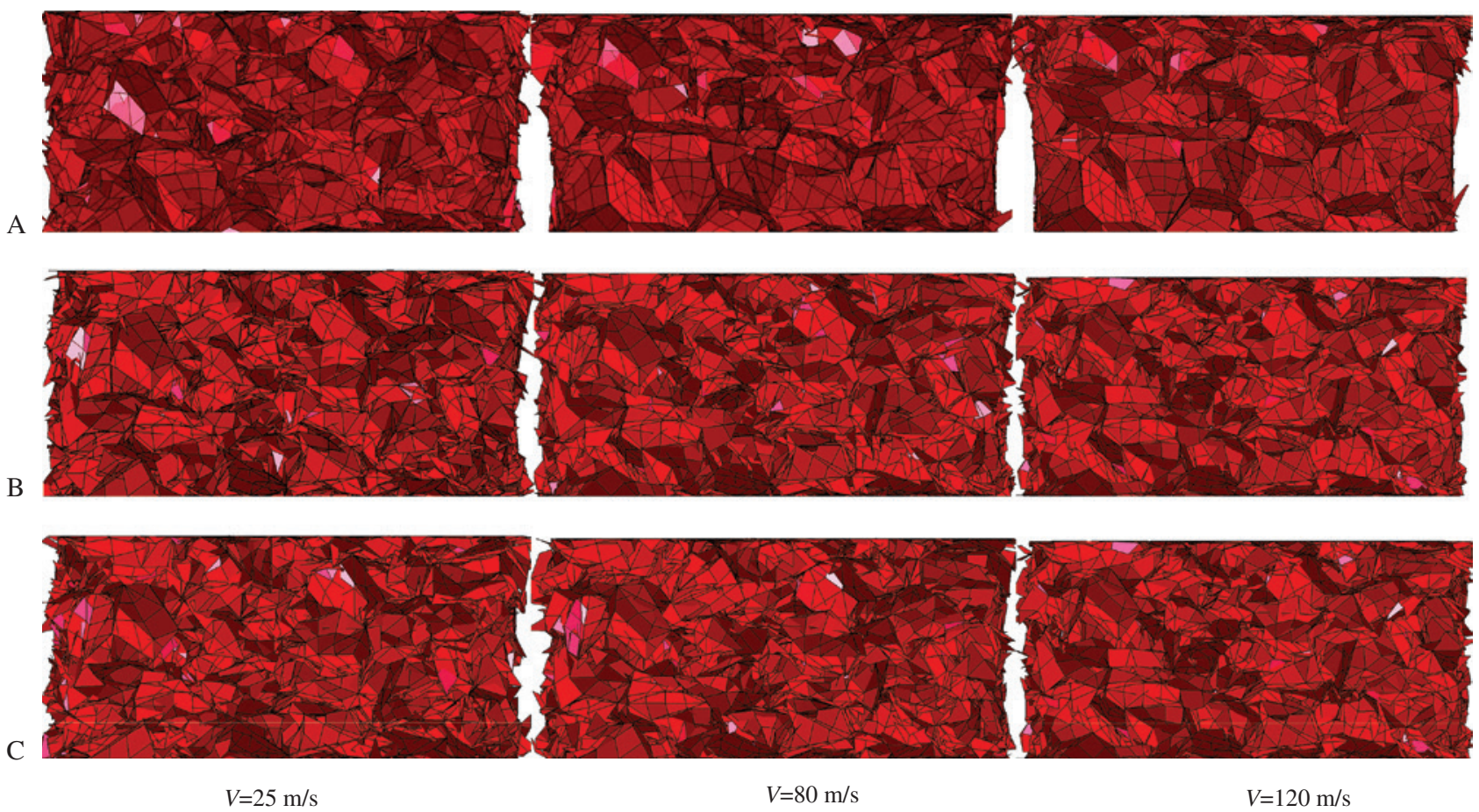

Figure 3: Deformation patterns $(\mathrm{A}, \bar{H}=0 ; \mathrm{B}, \bar{H}=0.03 ; \mathrm{C}, \bar{H}=0.07)$.

I-shape mode. But when introducing the strain hardening in Figure 3B and C, there is much difference. For example, for $\bar{H}=0.07$, the deformation bands are still randomly distributed at $V=120 \mathrm{~m} / \mathrm{s}$ compared with the I-shape collapse bands in Figure 3A at the same velocity, which indicates that the cell wall strain hardening may postpone the onset of the localized collapse bands. For further verification of this indication, the deformation modes are mapped in Figure 4. From the figure, there is a large delay of the

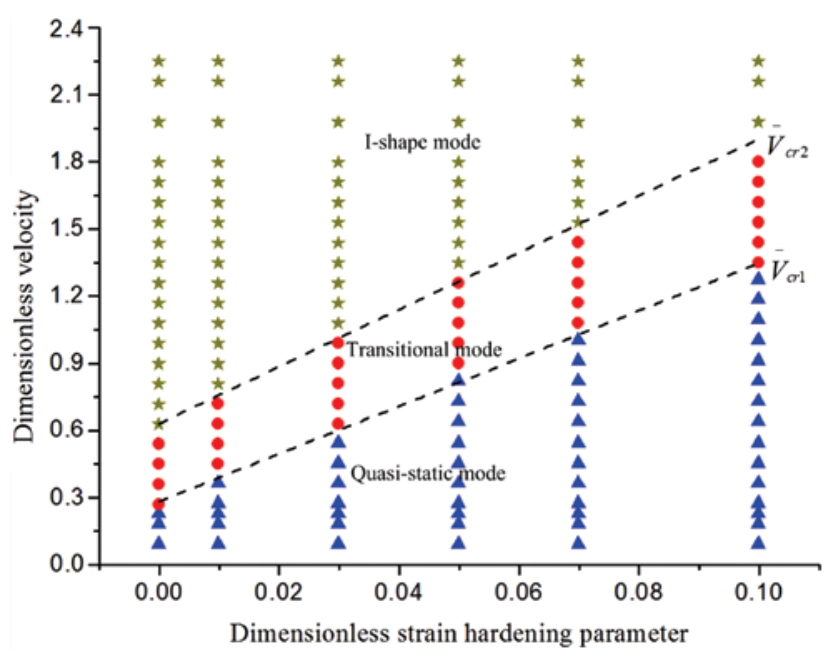

Figure 4: Deformation map of closed-cell foams with strain hardening. deformation mode transformation if the cell wall material strain hardening is incorporated in the foams. This is because the strain hardening of the cell wall material enhances the strength and stiffness of the foam cell; thus, this increases the capability of the deformation resistance and resistance destruction. The dimensionless critical velocity of deformation mode transformation approximates linearly and positively to the strain hardening parameter; this relation can be fitted and formulated as

$$
\bar{V}_{c r 1}=10.66 \bar{H}+0.28 \text { and } \bar{V}_{c r 2}=12.72 \bar{H}+0.62
$$

From another aspect, even though the strain hardening could retard the deformation transformation, if the impact velocity is sufficiently high, the foam will always collapse in the I-shape. In spite of increasing the hardening tangent modulus, localized deformation in the form of narrow crush bands dominates the deformation mode at a high impact velocity. This means that the effect of the strain hardening will be overwhelmed by higher impact velocity, and the deformation with material strain hardening will be the same with the elastic - perfect plastic case at last. In other words, high impact velocity could bring about a phenomenon - strain hardening convergence on the deformation of the closed-cell foams. This convergence phenomenon may play some role on the crushing stress and plastic energy dissipation, so this will be investigated below in detail. 


\subsection{Crushing stress}

The crushing stress and the nominal strain during the dynamic deformation of three-dimensional Voronoi model were calculated from the numerical simulations under different impact loadings. Here, the crushing stress, which was calculated at both the impact and the stationary sides, was defined as the reaction force at the rigid plate divided by the contact area between the rigid plate and the specimen. The nominal strain used in the simulation was set to be the total compression displacement of the specimen divided by the specimen height $L$.

Figure 5 plots the crushing stress-strain relationship at the impact side. From the figure, the cell wall material strain hardening could enhance the crushing stress at the impact side for a specific impact velocity. This is owing to the increasing capacity of deformation resistance and resistance destruction of cells with material strain hardening; as found in the deformation diagrams, the crushing stress will be improved. For a particular dimensionless strain hardening parameter, such as $\bar{H}=0.03$ at a low velocity $(v \leq 50 \mathrm{~m} / \mathrm{s})$, there is a difference of the curves with no strain hardening cell wall material; compared with the horizontal post-collapse plateau of the case $\bar{H}=0.0$, the post-yield curve of the case $\bar{H}=0.03$ does not have a flat plateau but rises with a hardening slope. The slopes of the curves are further enhanced for a higher strain hardening parameter. However, with the increase of impact velocities (Figure 5C and D), the enhanced slope of post-yield curves will be weakened, being inclined to a flat plateau apart from some fluctuations in Figure 5D. Figure 6 gives the variations of crushing stress at the stationary side. Similarly with the stress at the impact side, from Figure 6, the stress is also enhanced by the strain
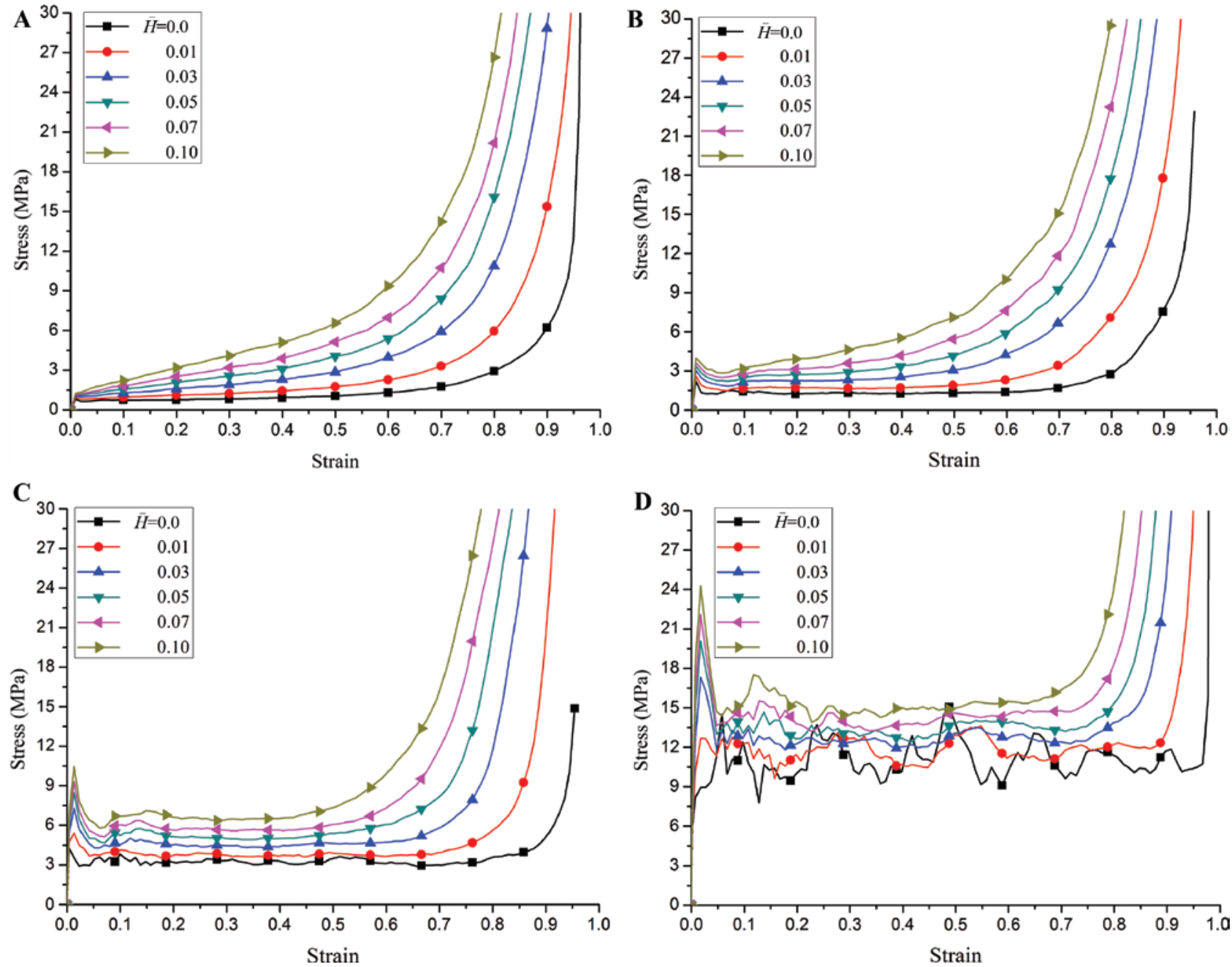

Figure 5: Crushing stress variation at the impact side $(A, 10 \mathrm{~m} / \mathrm{s} ; B, 50 \mathrm{~m} / \mathrm{s} ; \mathrm{C}, 120 \mathrm{~m} / \mathrm{s} ; \mathrm{D}, 250 \mathrm{~m} / \mathrm{s})$. 

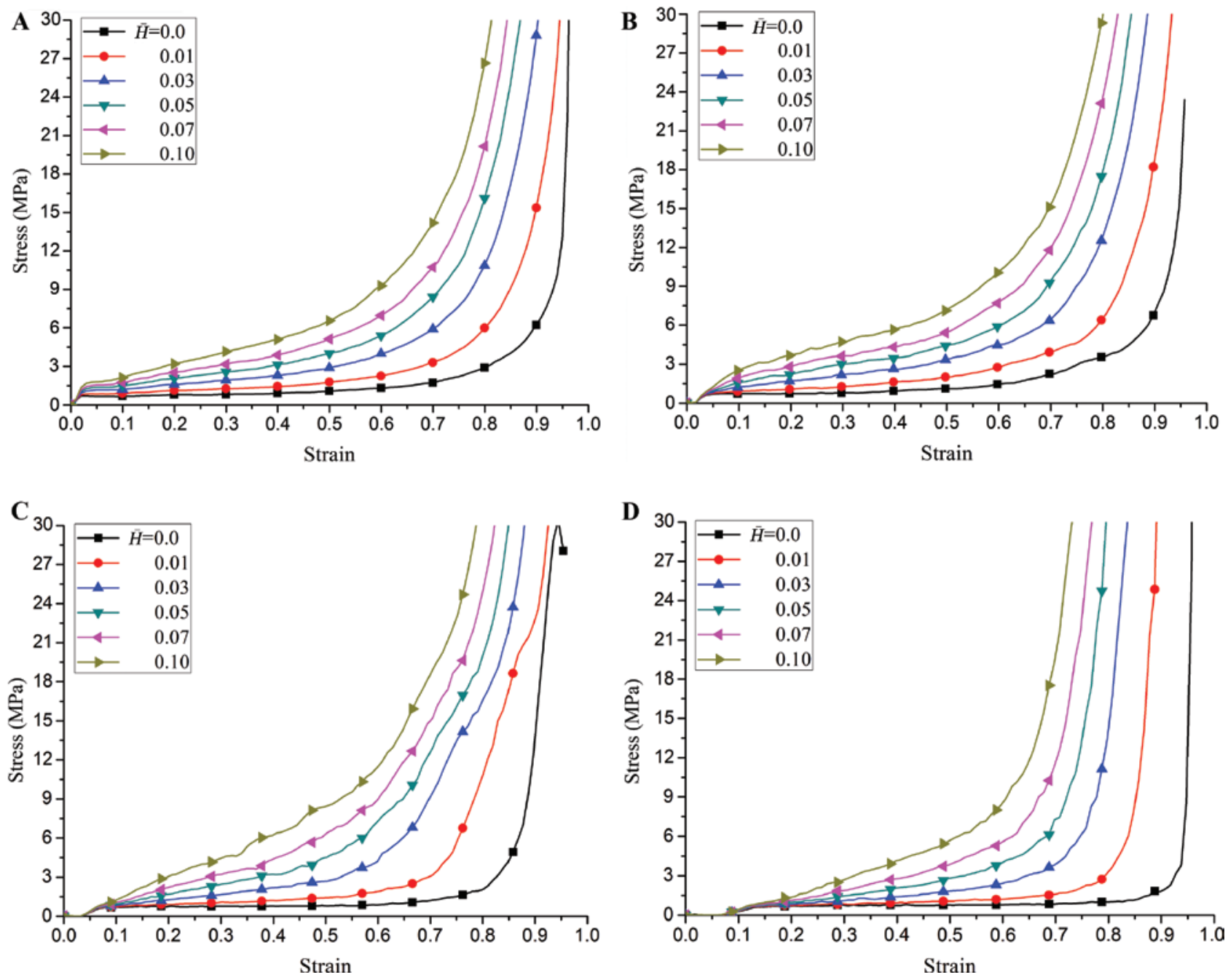

Figure 6: Crushing stress variation at the stationary side (A, $10 \mathrm{~m} / \mathrm{s} ; \mathrm{B}, 50 \mathrm{~m} / \mathrm{s} ; \mathrm{C}, 120 \mathrm{~m} / \mathrm{s} ; \mathrm{D}, 250 \mathrm{~m} / \mathrm{s})$.

hardening parameters at a certain impact velocity, but the stress maintains almost the same at different velocities; this insensitivity has also been observed in reference [21].

The plateau stress and densification strain are two crucial parameters to characterize the mechanical properties of cellular materials, so we also investigate them in the case of strain hardening. The energy efficiency-based approach was adopted to calculate the plateau stress and densification strain [28], as shown in Figure 7. Energy absorption efficiency was defined as

$$
\eta\left(\varepsilon_{a}\right)=\frac{\int_{\varepsilon_{c r}}^{\varepsilon_{a}} \sigma_{c}(\varepsilon) d \varepsilon}{\sigma_{c}(\varepsilon)_{\varepsilon=\varepsilon_{a}}}
$$

The plateau stress was determined by

$$
\sigma_{p}=\frac{\int_{\varepsilon_{c r}}^{\varepsilon_{a}} \sigma_{c}(\varepsilon) d \varepsilon}{\varepsilon_{a}-\varepsilon_{c r}}
$$

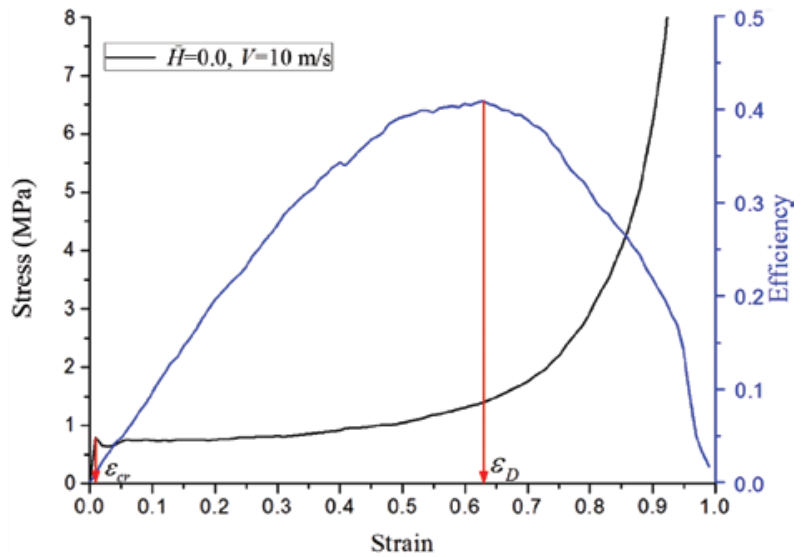

Figure 7: Capture of the densification strain.

Figure $8 \mathrm{~A}$ plots the plateau stress at the impact side; as is observed in Figure 5, the plateau stress is enhanced and positively correlated with the strain hardening parameter 

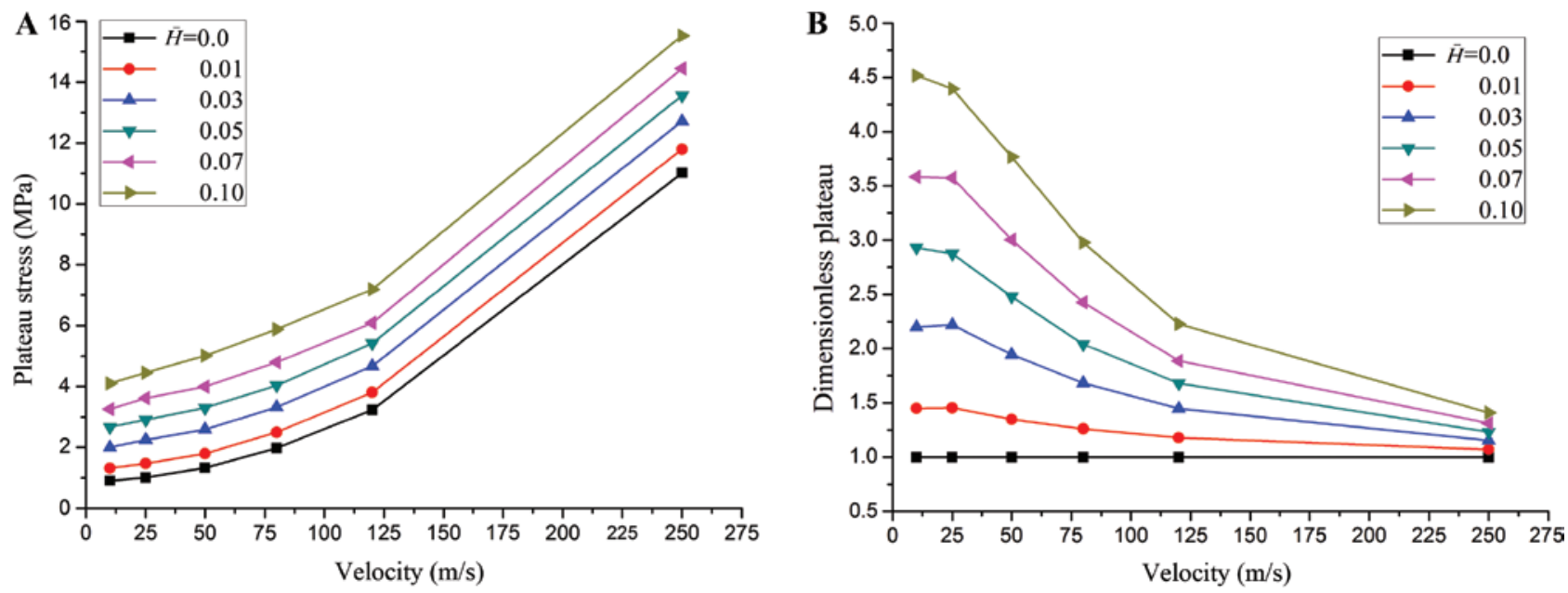

Figure 8: Plateau stress (A) and the dimensionless plateau (B) at the impact side.

as well as the impact velocity. However, it should be noted that the stress difference between different strain hardening parameters, such as between $\bar{H}=0.01$ and $\bar{H}=0.03$, remains almost constant across the range of the velocities considered in the study. This is to say that the enhancement of the plateau is mainly due to the impact velocity and secondarily because of strain hardening. It is similar to the observation in deformation mode transformation. For further understanding this, the dimensionless plateau is defined as

$$
\bar{\sigma}_{p}=\frac{\sigma_{p}(\bar{H})}{\sigma_{p}(\bar{H}=0)}
$$

Based on Figure $8 \mathrm{~A}$, the dimensionless data are replotted in Figure 8B. Evidently, there is a large deviation between the results of $\bar{H}=0$ and $\bar{H} \neq 0$ at a low impact velocity, and the dimensionless plateau gradually gets close to 1 as the impact velocity increases, which hints that there is a dimensionless plateau convergence, further confirming the strain hardening convergence; increasing the crushing velocity could diminish the effect of material strain hardening.

Figure 9A describes the densification strain at the impact side and shows that incorporating the material strain hardening could decrease the densification strain. The reason can be found in Figure 5; the post-yield curve with strain hardening does not have a flat plateau but rises with a hardening slope, and the slopes of the curves are further enhanced for a higher strain hardening parameter. Meanwhile, a higher impact velocity could enlarge the densification strain which could be interpreted by
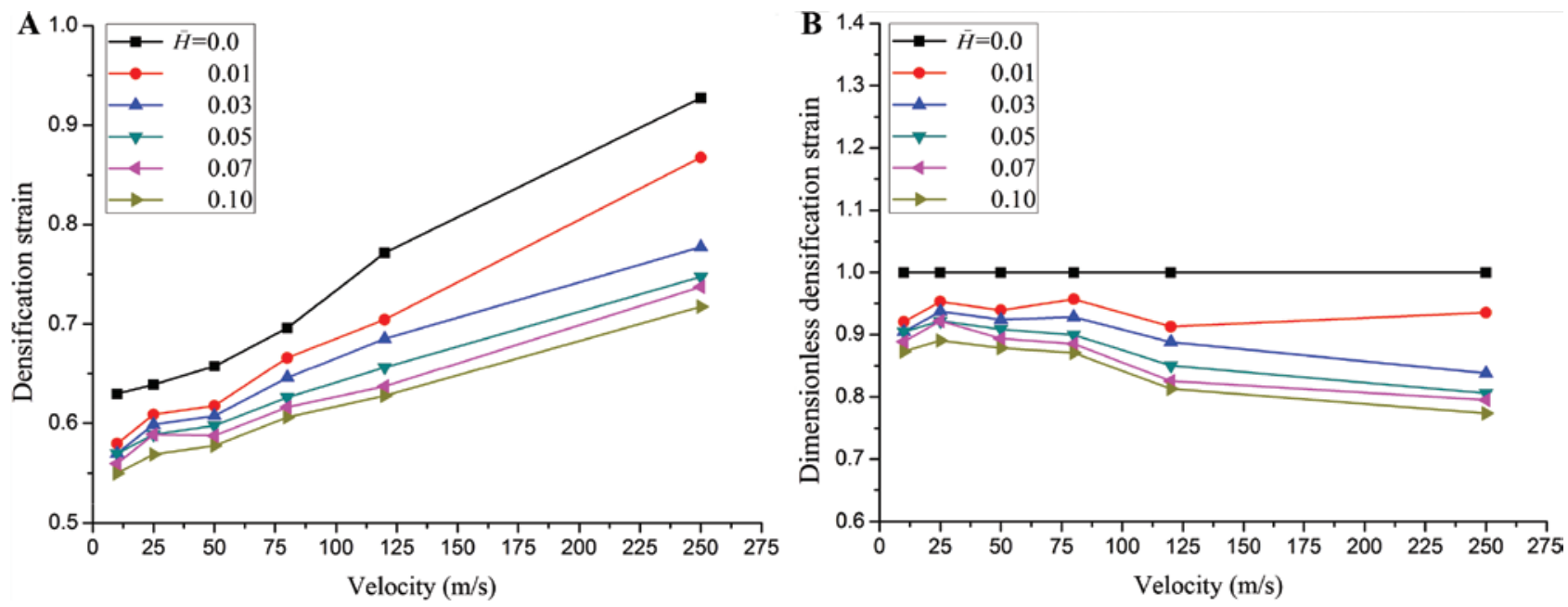

Figure 9: Densification strain (A) and the dimensionless one (B) at the impact side. 




Figure 10: Dimensionless plateau stress at the stationary side.

the deformation patterns in Figure 3; the crushing bands locally concentrate on the impact side at a higher impact velocity and propagate to the stationary side, so there will be a larger flat plateau. Here, a dimensionless parameter is also defined and expressed as

$$
\bar{\varepsilon}_{D}=\frac{\varepsilon_{D}(\bar{H})}{\varepsilon_{D}(\bar{H}=0)}
$$

The data are presented in Figure 9B; there is little variation of the results at different impact velocities, and no strain hardening convergence is observed in the dimensionless densification strain.

From Eq. (11), the dimensionless plateau stress at the stationary side is also calculated, as illustrated in Figure 10. The figure reveals that the material strain hardening could also strengthen the plateau at the stationary side, but for a certain strain hardening parameter, the plateau stress is insensitive to the impact velocity, which has been found in Figure 6. Moreover, the large difference with the plateau at the impact side is that there is no similar convergence for the dimensionless plateau at the stationary side, which is on account of this insensitivity of crushing stress found above, while from the aspect of the
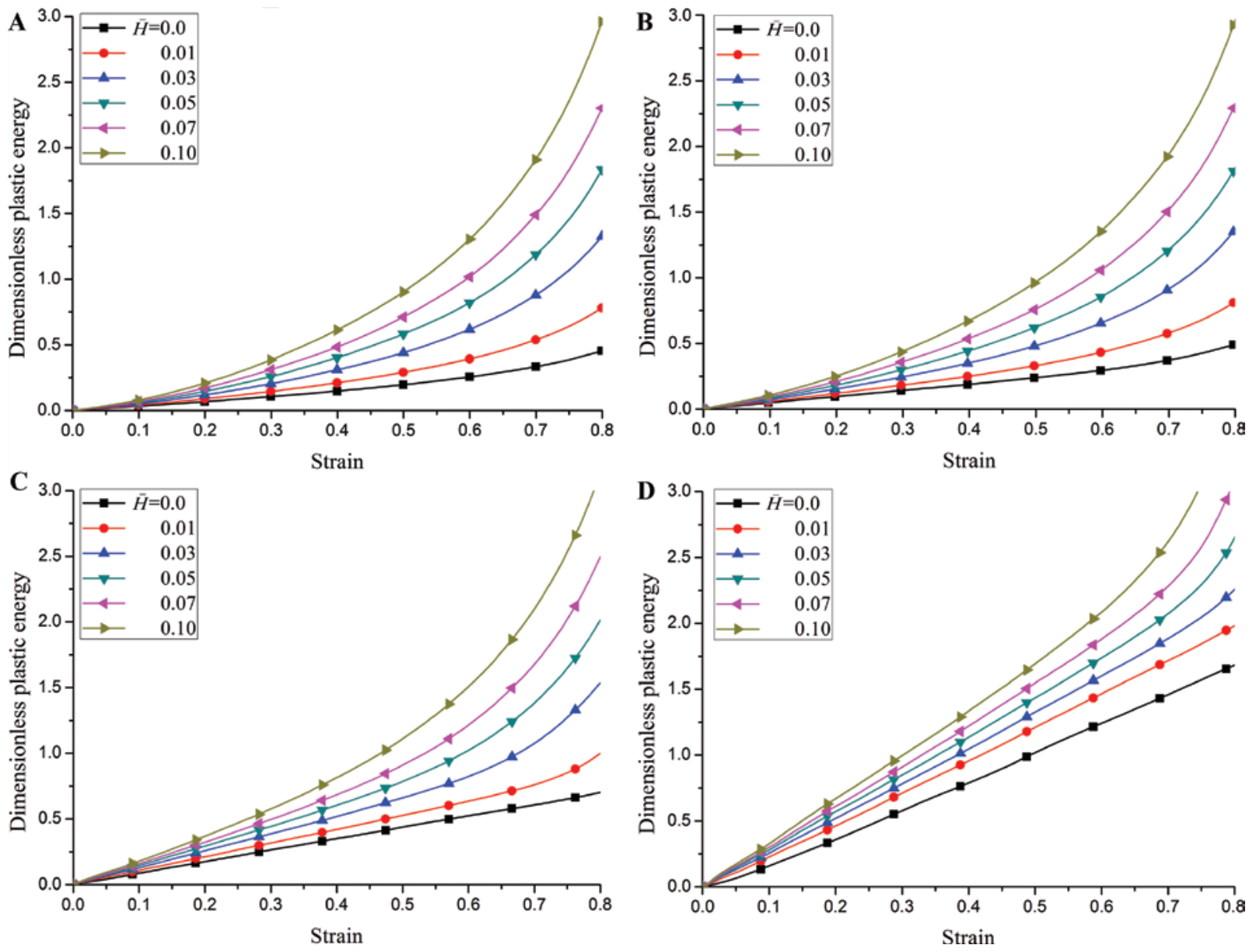

Figure 11: Dimensionless plastic energy dissipation at different velocities (A, $10 \mathrm{~m} / \mathrm{s} ; \mathrm{B}, 50 \mathrm{~m} / \mathrm{s} ; \mathrm{C}, 120 \mathrm{~m} / \mathrm{s} ; \mathrm{D}, 250 \mathrm{~m} / \mathrm{s})$. 
crushing stress at the impact side affected by the inertia effect, it is sensitive to the velocity which leads to the submergence of the strain hardening.

\subsection{Plastic energy dissipation}

Figure 11 reveals the variation of the dimensionless plastic energy dissipation at different velocities. From the figure, the plastic energy dissipation of models with strain hardening is always larger than the elasticperfectly plastic case across the crushing strain for a specific velocity. As mentioned above, this can be deciphered by the different deformations: material strain hardening can improve the strength and stiffness of the cells, which leads to higher deformation resistance; the plateau stress will be enhanced; and consequently, the performance of energy dissipation will also be strengthened. Another noticeable point is that the plastic energy with no strain hardening is almost linear with strain, while the curves with strain hardening are increasingly hardening, which is because there is a flat plateau stage of the elastic-perfectly plastic foam, while the post-yield stages of stress-strain curves with strain hardening rise with a hardening slope. As is observed in the aforementioned, increasing the impact velocity could weaken the effect of material strain hardening on the deformation and the crushing stress, so in order to investigate this phenomenon on the plastic energy dissipation, we doubly normalize the dimensionless plastic energy dissipation as follows:

$$
\bar{E}_{p p}=\frac{\bar{E}_{p}(\bar{H})}{\bar{E}_{p}(\bar{H}=0)}
$$

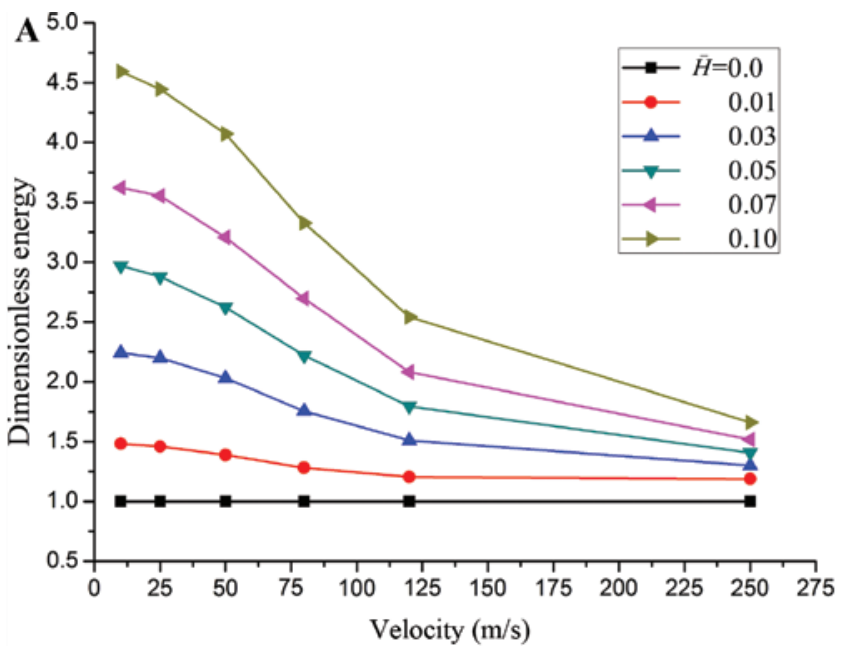

Figure 12 plots this doubly normalized parameter at 50\% and $70 \%$ crushing strain. At a low impact velocity, there is a large deviation of the double dimensionless energy between material strain hardening and elastic-perfect plastic cases, while all get close with a little deviation at $V=250 \mathrm{~m} / \mathrm{s}$. This further demonstrates that increasing the crushing velocity could fade the effects of material strain hardening. This confirms the strain hardening convergence effect on the plastic energy dissipation at a high impact velocity.

\section{Conclusions}

In the paper, three-dimensional Voronoi models were established to present the closed-cell foams. By considering the cell wall material strain hardening of foams, the simulations were carried out by using Ls-dyna 971 to investigate this effect on the dynamic responses of closedcell foams suffering from constant velocity impact.

In the aspect of the deformations, the results uncovered that there was a large delay of the deformation mode transformation if the cell wall material strain hardening was incorporated in the foams and the cell wall strain hardening could postpone the onset of the localized collapse bands. The strain hardening convergence phenomenon - the effect of the strain hardening would be overwhelmed by higher impact velocity - about the deformation was found in the closed-cell foams.

The crushing stress at both impact and stationary sides was taken into consideration. The cell wall material strain hardening could enhance the crushing stress at both sides for a specific impact velocity. At a moderate velocity,

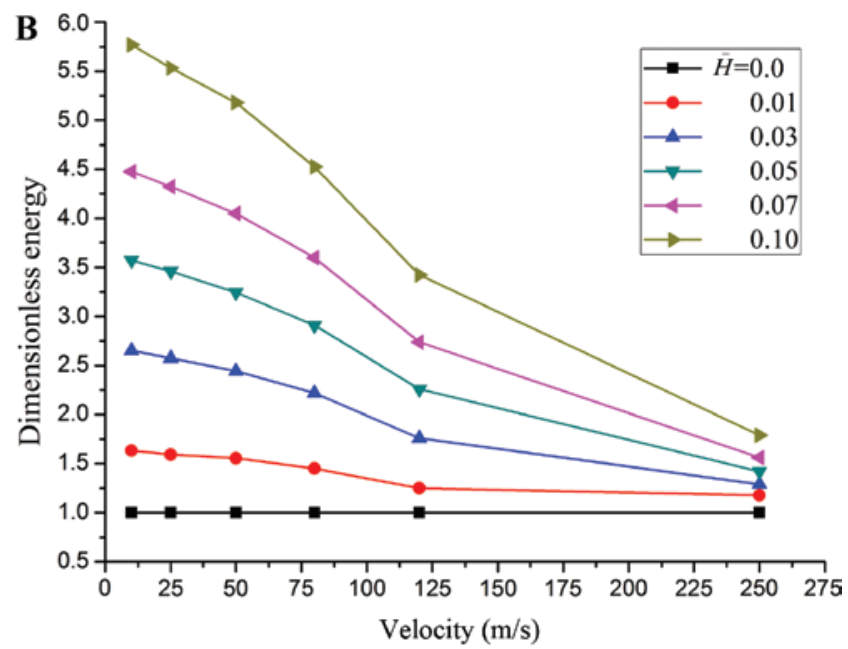

Figure 12: Doubly normalized plastic energy dissipation $\left(\bar{E}_{p p}\right)$ at $50 \%(\mathrm{~A})$ and $70 \%(\mathrm{~B})$ crushing strain, respectively. 
the post-yield curve with strain hardening did not have a flat plateau but had a hardening slope. The slopes of the curves were further enhanced for a higher strain hardening parameter. As the impact velocity increased, the enhanced slope of post-yield curves would be weakened, being inclined to a flat plateau. Meanwhile, the plateau stress and densification strain were also calculated. It was found that there was a dimensionless plateau convergence at the impact side, further confirming the strain hardening convergence. Material strain hardening could decrease the densification strain, but no similar convergence was observed.

Concerning the plastic energy dissipation, it could be always enhanced across the crushing strain if considering the material strain hardening because of a higher stress plateau. The plastic energy with no strain hardening was almost linear with strain while the curves with strain hardening were increasingly hardening. The strain hardening convergence also occurred on the plastic energy dissipation.

Acknowledgments: This work is supported by the National Natural Science Foundation of China (Grant No. 11172196), Shanxi Scholarship Council of China (2013-046), the Top Young Academic Leaders of Shanxi, the Outstanding Innovative Teams of Higher Learning Institutions of Shanxi and the Graduate student innovation Fund of TYUT (B2014025). The financial contributions are gratefully acknowledged.

\section{References}

[1] Gibson LJ, Ashby MF. Cellular Solids: Structures and Properties, 2nd ed., Cambridge University Press: UK, 1997.

[2] Li K, Gao XL. Int. J. Solids Struct. 2005, 42, 1777-1795.

[3] Jin T, Zhou ZW, Liu ZG, Shu XF. Sci. Eng. Compos. Mater. 2016, 23, 311-317.
[4] Wang ZH, Shen JH, Lu GX, Zhao LM. Mater. Sci. Eng. A 2011, 528, 2326-2330.

[5] Chen C, Lu TJ, Fleck NA. J. Mech. Phys. Solids 1999, 47, 2235-2272.

[6] Ruan D, Lu G, Wang B, Yu TX. Int. J. Impact Eng. 2003, 28, 161-182.

[7] Tan PJ, Reid SR, Harrigan JJ, Zou Z, Li S. J. Mech. Phys. Solids 2005, 53, 2206-2230.

[8] Zhu F, Chou CC, Yang KH. Composites: Part B 2011, 42, 1202-1211.

[9] Silva MJ, Hayes WC, Gibson LJ. Int. J. Mech. Sci. 1995, 37, 1161-1177.

[10] Papka SD, Kyriakides S. J. Mech. Phys. Solids 1994, 42, 1499-1532.

[11] Marchi CS, Despois JF, Mortensen A. Acta Mater. 2004, 52, 2895-2902.

[12] Marchi CS, Mortensen A. Acta Mater. 2001, 49, 3959-3969.

[13] Amsterdam E, De Hosson JTM, Onck PR. Scripta Mater. 2008, 59, 653-656.

[14] Despois JF, Mueller R, Mortensen A. Acta Mater. 2004, 54, 4129-4142.

[15] Mangipudi KR, van Buuren SW, Onck PR. Int. J. Solids Struct. 2010, 47, 2081-2096.

[16] Mousanezhad D, Ghosh R, Ajdari A, Hamouda AMS, NayebHashemi H, Vaziri A. Int. J. Mech. Sci. 2014, 89, 413-422.

[17] Song YZ, Wang ZH, Zhao LM, Luo J. Mater. Design 2010, 31, 4281-4289.

[18] Mahin KW, Hanson K, Morris JW. Acta Mater. 1980, 28, 443-453.

[19] Weaire D, Fortes AM. Adv. Phys. 1994, 43, 685-738.

[20] Gan YX, Chen C, Shen YP. Int. J. Solids Struct. 2005, 42, 6628-6642.

[21] Li ZQ, Zhang JJ, Fan JH, Wang ZH, Zhao LM. Mech. Mater. 2014, $68,85-94$.

[22] Li K, Gao XL, Wang J. Int. J. Solids Struct. 2007, 44, 5003-5026.

[23] Zheng Z], Yu JL, Li JR. Int. J. Impact Eng. 2005, 32, 650-664.

[24] Ma GW, Ye ZQ, Shao ZS. Int. J. Impact Eng. 2009, 36, 775-782.

[25] Shen CJ, Lu G, Yu TX. Compos. Struct. 2013, 98, 282-293.

[26] Zheng Z], Liu YD, Yu JL, Reid SR. Int. J. Impact Eng. 2012, 42, 66-79.

[27] Liu YD, Yu JL, Zheng Z], Li JR. Int. J. Solids Struct. 2009, 46, 3988-3998.

[28] Li QM, Magkiriadis I, Harrigan JJ. J. Cell. Plast. 2006, 42, 371-392. 